\title{
Correction to: Computed tomography surveillance helps tracking COVID-19 outbreak
}

\author{
Akihiro Machitori $^{1} \cdot$ Tomoyuki Noguchi $^{2,3,4}$ (1) $\cdot$ Yusuke Kawata $^{1} \cdot$ Nobuhiko Horioka $^{5} \cdot$ Akihiro Nishie $^{6}$. \\ Daisuke Kakihara $^{6} \cdot$ Kousei Ishigami $^{6} \cdot$ Shigeki Aoki $^{7} \cdot$ Yutaka Imai $^{8}$
}

Published online: 27 August 2020

(c) Japan Radiological Society 2020

\section{Correction to: Japanese Journal of Radiology https://doi.org/10.1007/s11604-020-01026-z}

The authors would like to correct Abstract and text of original publication as follows:

\section{Correction in Abstract \\ Original publication:}

Their PCR test results were positive $(n=62.5-398 \%)$, negative $(n=8.9-57 \%)$, unknown $(n=26.2-167 \%)$, and other disease $(n=2.4-15 \%)$.

The original article can be found online at https://doi.org/10.1007/ s11604-020-01026-z.

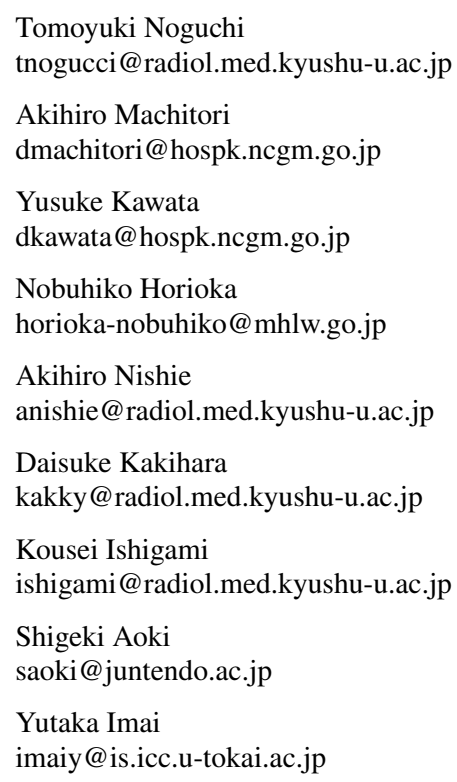

\section{Corrected sentence:}

Their PCR test results were positive $(n=398,62.5 \%)$, negative $(n=57,8.9 \%)$, unknown $(n=167,26.2 \%)$, and other disease $(n=15,2.4 \%)$.

\section{Correction in text (in Results section) Original publication:}

The most common CT findings judged by radiologists were bilateral lung (male: female $=78.2-82.4 \%$ ) and distributed GGOs (male: female $=80.8-83.0 \%$ ). The crazy-paving pattern (male: female $=41.0-43.1 \%$ ) and consolidation
1 Department of Radiology, National Center for Global Health and Medicine, Kohnodai Hospital, 1-7-1 Kohnodai, Ichikawa City, Chiba Province 272-8516, Japan

2 Department of Clinical Research, Center for Clinical Sciences, National Center for Global Health and Medicine, 1-21-1 Toyama, Shinjuku-ku, Tokyo 162-8655, Japan

3 Department of Radiology, National Hospital Organization Kyushu Medical Center, 1-8-1 Jigyohama, Chuo-ku, Fukuoka City, Fukuoka Province, Japan

4 Department of Clinical Research, National Hospital Organization Kyushu Medical Center, 1-8-1 Jigyohama, Chuo-ku, Fukuoka City, Fukuoka Province 810-8563, Japan

5 General Affairs Division, Health Policy Bureau, Ministry of Health, Labour and Welfare, 1-2-2 Kasumigaseki, Chiyoda-ku, Tokyo 100-8916, Japan

6 Department of Clinical Radiology, Graduate School of Medical Sciences, Kyushu University, 3-1-1 Maidashi, Higashi-ku, Fukuoka City, Fukuoka Province 812-8582, Japan

7 Department of Radiology, Juntendo University, 2-1-1 Hongo, Bunkyo-ku, Tokyo 113-8421, Japan

8 Department of Radiology, Tokai University Hachioji Hospital, 1838 Ishikawa-cho, Hachioji City, Tokyo 192-0032, Japan 
(male: female $=33.2-35.0 \%$ ) were observed at about onehalf the frequency of GGOs (Fig. 4).

\section{Corrected sentence:}

The most common CT findings judged by radiologists were bilateral lung (male: female $=78.2 \%: 82.4 \%$ ) and distributed GGOs (male: female $=80.8 \%: 83.0 \%$ ). The crazypaving pattern (male: female $=41.0 \%: 43.1 \%$ ) and consolidation (male: female $=33.2 \%: 35.0 \%$ ) were observed at about one-half the frequency of GGOs (Fig. 4).

\section{Correction in figure legend of Fig. 5}

\section{Original publication:}

Significant differences between the PCR-positive and -negative patients in CT-surveillance were observed in the bilateral lung involvement (70.2-84.2\%) and the consolidation (33.9-50.9\%). In the meantime, GGOs (83.5-83.9\%) and the crazy-paving pattern (36.8-44.5\%) were commonly observed

\section{Corrected sentence:}

Significant differences between the PCR-positive and -negative patients in CT-surveillance were observed in the bilateral lung involvement $(70.2 \%, 84.2 \%)$ and the consolidation $(33.9 \%, 50.9 \%)$. In the meantime, GGOs $(83.5 \%$, $83.9 \%)$ and the crazy-paving pattern $(36.8 \%, 44.5 \%)$ were commonly observed

Publisher's Note Springer Nature remains neutral with regard to jurisdictional claims in published maps and institutional affiliations. 